\title{
Successful Second Trimester Myomectomy in a Critically III Patient
}

\author{
Farshad Tahmasebi ${ }^{1 *}$, Mridula Morje ${ }^{1}$, Alice Hurrell${ }^{2}$, Charles Gallaher ${ }^{3}$ and Bashir Dawlatly ${ }^{2}$ \\ ${ }^{1}$ Obstetrics and Gynaecology, St Thomas' Hospital, London, UK \\ ${ }^{2}$ Obstetrics and Gynaecology, Whipps Cross University Hospital, UK \\ ${ }^{3}$ Emergency Medicine, St Thomas' Hospital, London, UK
}

${ }^{*}$ Corresponding author: Farshad Tahmasebi, Post-CCT Gynae Oncology Fellow, St Thomas' Hospital, London, Flat 20 Charing Cross Mansions, 26 Charing Cross Road, WC2H Odg, London, UK, E-mail: tfarshad@hotmail.com

Received date: November 30, 2017; Accepted date: June 02, 2018; Published date: June 08, 2018

Copyright: (C) 2018 Tahmasebi F, et al. This is an open-access article distributed under the terms of the Creative Commons Attribution License, which permits unrestricted use, distribution, and reproduction in any medium, provided the original author and source are credited.

Citation: Tahmasebi F, Morje M, Hurrell A, Gallaher C, Dawlatly B (2018) Successful Second Trimester Myomectomy in a Critically III Patient. Crit Care Obst Gyne. Vol.4 No.2:5.

\section{Abstract}

Uterine leiomyomas (myomas) poses antenatal, intrapartum and postnatal risks, including pregnancy loss, preterm delivery, antepartum and postpartum haemorrhage, abnormal placental implantation, fetal malpresentation, mechanical dystocia and need for caesarean section.

Though traditionally contraindicated due to their risk of pregnancy loss and massive haemorrhage, antenatal myomectomies have been shown to be beneficial in selected cases in more recent studies.

We present a case of a patient who underwent a successful open subserosal myomectomy at 24 weeks gestation for worsening pelvic pain and subacute bowel obstruction. Following a turbulent post-operative phase and admission to the critical care unit, the pregnancy continued without further complications. At 38 weeks gestation, the patient underwent an uncomplicated elective caesarean section.

\section{Keywords: Myomectomy; Pregnancy; Antenatal; Critical} care

\section{Introduction}

With increasing age of obstetric patients, the prevalence of myomas in pregnancy has risen to $2-10 \%$. Of these, $10-30 \%$ develops complications [1].

The number, size and location of the myomas often contribute to the type and severity of symptoms. Specifically, retroplacental myomas and myomas larger than $3.6 \mathrm{~cm}$ have been associated with higher incidence of complications including pressure symptoms, torsion, degeneration, abnormal placentation, pregnancy loss, fetal growth restriction and malpresentation [2].

Simple analgesia and a short course of non-steroidal antiinflammatory drugs tend to be the mainstay of treatment.
Surgical management has been described in case reports and retrospective studies [3-12]. The majority of these surgeries were performed in the second trimester for intractable pelvic pain and had excellent outcomes: very few pregnancy losses and no hysterectomies have been reported. Traditional recommendations, including operating exclusively on pedunculated myomas [2], only during the fourth and sixth month of pregnancy [13] or only during the 14th to 15th week, warrant re-evaluation [14].

Literature focusing on outcomes of myomectomies performed during the first trimester is limited $[10,15]$.

\section{Case}

A 31-year-old Afro-Caribbean primigravid woman presented to hospital at 22 weeks gestation with acute generalised abdominal pain, abdominal distension, vomiting and constipation. Abdominal examination found the uterus to be tender, irregular and larger than expected for gestational age. The fetal heartbeat was normal. Vital signs showed a respiratory rate of 30 breaths per minute (breaths/min), oxygen saturations (SpO2) of $97 \%$ in air, heart rate of 150 beats per minute (bpm), blood pressure of $138 / 88 \mathrm{mmHg}$, and a normal temperature.

Urine dipstick was positive for ketones +++ and protein +. Admission blood tests showed a total white cell count (WBC) of $20.9 \times 109 / \mathrm{L}$ (normal range: 4.0-11.0 $\times 109 / \mathrm{L}$ ), haemoglobin $(\mathrm{Hb})$ $91 \mathrm{~g} / \mathrm{L}(120-150 \mathrm{~g} / \mathrm{L})$, platelets $896 \times 109 / \mathrm{L}(100-400 \times 109 / \mathrm{L})$, creatinine $124 \mu \mathrm{mol} / \mathrm{L}(45-84 \mu \mathrm{mol} / \mathrm{L})$, and lactate $2.4 \mathrm{mmol} / \mathrm{L}$ (0.4-2.2 mmol/L).

The patient was made nil-by-mouth and treated for sepsis and possible bowel obstruction with analgesia, antibiotics, intravenous fluids and prophylactic-dose low molecular weight heparin.

An ultrasound scan showed an appropriately grown singleton fetus and a subserous fundal leiomyoma, measuring $17 \times 10 \mathrm{~cm}$, with free fluid in the abdomen.

An urgent MRI scan was performed and this confirmed the presence of a $20 \mathrm{~cm}$ subserosal fibroid with large areas of cystic 
degeneration and necrosis. The fibroid had grown significantly during the preceding months and it had recently degenerated, as compared to a recent ultrasound scan. Beneath the umbilicus, there was another $9 \mathrm{~cm}$ subserosal fibroid with cystic degeneration/necrosis, which had similarly enlarged and degenerated. The MRI scan also showed a significant amount of free fluid within the abdomen, approximately $1000 \mathrm{~mL}$. The aetiology of this free fluid was uncertain, but it was suggested that the fibroid might have ruptured into the abdomen.

The patient underwent ultrasound-guided ascitic tap which showed a sterile exudate.

Though blood cultures were negative, the urine culture grew Escherichia coli. Intravenous antibiotics were continued.

On day 6 of admission, blood tests showed an improvement, with decreasing inflammatory markers (WBC $13.0 \times 109 / \mathrm{L}$ from $20.9 \times 109 / \mathrm{L}$ ) and improving renal function (creatinine 77 $\mu \mathrm{mol} / \mathrm{L})$.

By day 11, the patient was stable enough for transfer to a tertiary centre with a level three neonatal unit.

Despite on-going treatment with analgesia and antibiotics, she remained systemically unwell with sepsis and severe abdominal pain.

On day 13 , at gestational age of $23^{+6}$, the patient's condition deteriorated, with increasing abdominal distension. Vital signs revealed a respiratory rate of 24 breaths/min, heart rate 134 bpm, and blood pressure $130 / 88 \mathrm{mmHg}$. Bloods tests were as follows: Hb $87 \mathrm{~g} / \mathrm{L}$ (booking Hb $113 \mathrm{~g} / \mathrm{L}$ ), WBC $16.5 \times 109 / \mathrm{L}$, platelets $939 \times 109 / \mathrm{L}$, C-reactive protein $153 \mathrm{mg} / \mathrm{L}(0-4 \mathrm{mg} / \mathrm{L})$, albumin $27 \mathrm{mmol} / \mathrm{L}(40-52 \mathrm{~g} / \mathrm{L})$, lactate $0.9 \mathrm{mmol} / \mathrm{L}$, liver and renal function normal.

Two doses of steroid were administered to aid fetal lung maturation. In view of the lack of improvement with conservative measures, it was recommended that she undergo a laparotomy and myomectomy to remove the myomas. She was thoroughly counselled about the risks of uterine surgery at this advanced gestation, including the risk of pregnancy loss.

The patient proceeded to open myomectomy, with interventional radiology cover via internal iliac artery catheters. Surgical access was via midline laparotomy incision and two large fibroids were removed.

The patient had a stormy post-operative course, complicated by Serratia septicaemia, anaemia requiring blood transfusion, pain and poor mobility due in part to significant oedema.

She gradually recovered over the course of six weeks, and was discharged fit and well with an appropriately developing fetus. She went on to deliver by elective caesarean section at 38 weeks gestation.

\section{Discussion}

\section{Antenatal myomectomies}

The role of surgery in the antenatal management of myomas remains a controversial topic.
Though the majority of pregnant patients with myomas remain asymptomatic, $10-30 \%$ of them exhibit complications of varying characteristics and severity, depending on the site, size and number of myomas [1].

Complications arise in the form of pressure symptoms, red degeneration, torsion, and adverse pregnancy outcomes including mechanical dystocia, late miscarriage, preterm delivery, and antepartum and postpartum haemorrhage $[1,3,10,16]$.

In 2004, Sheiner et al. reported increased risk of adverse pregnancy outcomes in women with uterine myomas, including caesarean section (odds ratio $[O R]=6.7$ ), placental abruption $(\mathrm{OR}=2.6)$ and preterm delivery $(\mathrm{OR}=1.4)$ [17].

Expectant management is the mainstay of treatment due to the potential risks associated with surgery, including pregnancy loss during uterine manipulation and haemorrhagic complications from engorged vasculature [18].

However, more recent studies have shown myomectomy for subserous and pedunculated fibroids to be safe during the second trimester, especially with detailed pre-operative imaging $[5,9,12]$. In selected patients, antenatal myomectomies have even posed better outcomes $[10,15]$.

Indications for surgical intervention include pain refractory to medical management, clinical deterioration with haemodynamic instability, and suspected malignancy $[1,9,12,19]$. In recent years, laparoscopic management has been preferred over open surgery owing to better visualisation of viscera following intraabdominal insufflation and speedier recovery postoperatively $[1,11]$.

Though no formal guidelines exist on laparoscopic myomectomies in pregnancy, surgical recommendations have been provided through case reports. These include trocar insertion using Hasson technique, gradual intra-abdominal insufflation and exsufflation, and minimal uterine manipulation. Additionally, the fetal heart should be auscultated pre- and postoperatively $[4,18]$

Saccardi et al. performed a laparoscopic myomectomy at 15 weeks for a $24 \mathrm{~cm}$ pedunculated fibroid that was causing severe pain and constipation [3]. The patient had an uneventful antenatal period following the procedure, but required an emergency caesarean section for fetal distress. A similar case was described in a 10 weeks pregnant patient by Kosmidis et al. [4].

Our patient required a laparotomy because of worsening sepsis, and the number and location of her fibroids.

Recent case reports on patients undergoing open antenatal myomectomy include Shafiee et al. who performed an open myomectomy at 22 weeks gestation in Indonesia for a degenerative fibroid that was causing severe pain resistant to medical therapy [5]. To limit blood loss, they occluded both uterine arteries at the base of the broad ligaments using Foley catheters but released the clamps every eight minutes to allow fetal oxygenation. The patient had an uncomplicated elective Caesarean section at 38 weeks gestation. 
Kasum described a case where a highly vascular subserosal fibroid caused a massive haemoperitoneum at 15 weeks gestation, mandating an emergency laparotomy [6]. The rest of the pregnancy was uneventful.

Jhalta et al. performed a laparotomy on a patient at 14 weeks gestation for a $16 \mathrm{~cm}$ degenerate fibroid that could not be differentiated from an ovarian mass on ultrasonography [7]. Postoperatively, the patient was given tocolytics and progesterone, and delivered vaginally at term.

A similar case was described by Umezurike et al. where a 32 $\mathrm{cm}$ subserous fibroid had undergone cystic degeneration, and was removed at laparotomy [8]. Radiologically, the fibroid had appeared similar to an ovarian tumour.

There is very limited data on the safety and efficacy of first trimester procedures. Carolis et al. reported four women who underwent myomectomies in the first trimester: three had a rapidly growing pelvic mass, and one had persistent pain [9] One patient was lost to follow up, but the remaining three patients had uncomplicated caesarean sections at term.

\section{Admission to critical care unit}

Our patient was unwell enough to require admission to the critical care unit. The approach to the management of critical illness during pregnancy involves the application of usual critical care principles, modified where necessary to account for the physiological (and pathological) changes seen in pregnancy [20]. The principles of managing the critically ill pregnant patient can be considered according to an ABCDE approach.

\section{Airway}

A number of factors make endotracheal intubation of the obstetric patient more challenging than non-pregnant controls, and some of these challenges are compounded by critical illness. Decreased functional residual capacity and increased oxygen consumption both contribute to rapid desaturation of the obstetric patient on induction of anaesthesia, as does critical illness in general $[21,22]$.

\section{Breathing}

Most aspects of mechanical ventilation are similar in obstetric and non-obstetric patients. Target partial pressure of arterial carbon dioxide $\left(\mathrm{PaCO}_{2}\right)$ may be set lower (4.00-4.27 kPa) to replicate the physiological respiratory alkalosis which occurs in pregnancy [23], thus minimising fetal acidosis, though this target may be relaxed in to $6.00 \mathrm{kPa}$ in lung-protective ventilation. Optimal oxygen saturation is not known; to prevent fetal hypoxia whilst minimising lung trauma from aggressive ventilation, a target of $95 \%$ is considered reasonable [24].

\section{Circulation}

A crucial difference between obstetric and non-obstetric patients with respect to circulation is the need for maintenance of left lateral tilt beyond 20 weeks gestation [25], particularly as inferior vena cava compression by the gravid uterus may be asymptomatic, and any limitation of uteroplacental blood flow is to be avoided20. Despite varying degrees of placental transfer and effects on uterine tone, a number of vasopressors/inotropes are considered relatively safe in pregnancy-certainly safer than permitting maternal and fetal hypotension-and these drugs should be used in patients who fail to respond to early, aggressive volume resuscitation [26].

\section{Disability}

The appropriateness of common sedatives used in nonpregnant ICU patients is variable in the obstetric population. Propofol is a US Food and Drug Administration (FDA) category $B$ drug (i.e. animal studies have failed to demonstrate a risk to the fetus); morphine and fentanyl are category $C$ drugs (i.e. animal reproduction studies have shown an adverse effect on the fetus, but potential benefits may warrant use of the drug in pregnant women despite potential risks); midazolam and lorazepam are category $D$ drugs (i.e. there is positive evidence of human fetal risk, but potential benefits may warrant use of the drug in pregnant women despite potential risks), and are generally considered contraindicated in pregnancy [20].

\section{Exposure}

Critically ill pregnant patients should be monitored as per non-pregnant ICU patients, with the addition of fetal monitoring depending on the gestational age and clinical scenario. Monitoring the fetus can help in determining the adequacy of maternal respiratory and cardiovascular parameters [20].

Our patient had severe intractable pain and symptoms of subacute bowel obstruction. After failing to improve with conservative management and after thorough counselling, she opted for an open myomectomy in the second trimester. This decision was made after reviewing the scant literature available on both first and second trimester myomectomies. Despite a stormy post-operative recovery, she went on to deliver a wellgrown healthy infant at term.

\section{Conclusion}

Various case reports over the last few years have demonstrated that with detailed imaging and careful preoperative mapping and risk assessment, antenatal myomectomy is beneficial in certain patients. All patients should be carefully counselled on an individual basis.

As the patients requiring surgery are often clinically unwell and refractory to medical management, multidisciplinary advice must be sought including involvement of senior medical and anaesthetic staff.

Our experience adds to the growing body of evidence and provides reassurance to pregnant women with uterine myomas: in select cases, experienced surgeons can successfully perform myomectomies during pregnancy. These patients may achieve a successful pregnancy outcome, as in our case, where the myomectomy relieved the patient's symptoms and led to her delivering a healthy baby at 38 weeks gestation. 


\section{Author Contributions}

$\mathrm{BD}$ and FT were involved in conception of this case report and literature search following the care of the involved patient and prompting the paper to be created. $\mathrm{AH}$ and $\mathrm{FT}$ were involved in data collection for the case report, which was written by AH. FT, MM and CG performed the literature review. FT, MM and CG edited the case report and literature review and wrote the introduction, as well as creating the initial draft of the article. Final approval of the article was a joint contribution between all listed authors.

\section{References}

1. Milazzo GN, Catalano A, Badia V, Mallozzi M, Caserta D (2017) Myoma and myomectomy: Poor evidence concern in pregnancy. J Obstet Gynaecol Res 43: 1789-1803.

2. Suwandinata FS, Gruessner SEM, Omwandho COA, Tinneberg HR (2008) Pregnancy-preserving myomectomy: preliminary report on a new surgical technique. Eur J Contracept Reprod Health Care 13: 323-326.

3. Saccardi C, Visentin S, Noventa M, Cosmi E, Litta P, et al. (2014) Uncertainties about laparoscopic myomectomy during pregnancy: A lack of evidence or an inherited misconception? A critical literature review starting from a peculiar case. Minim Invasive Ther Allied Technol 24: 189-194.

4. Kosmidis C, Pantos G, Efthimiadis C, Gkoutziomitrou I, Georgakoudi E, et al. (2015) Laparoscopic excision of a pedunculated uterine leiomyoma in torsion as a cause of acute abdomen at 10 weeks of pregnancy. Am J Case Rep 16: 505-508.

5. Shafiee MN, Nor Azlin MI, Arifuddin D (2012) A Successful Antenatal Myomectomy. Malays Fam Physician 7: 42-45.

6. Kasum M (2010) Hemoperitoneum caused by a bleeding myoma in pregnancy. Acta Clin Croat 49: 197-200.

7. Jhalta P, Negi SG, Sharma V (2016) Successful myomectomy in early pregnancy for a large asymptomatic uterine myoma: case report. Pan Afr Med J 24: 228.

8. Umezurike C, Feyi-Waboso P (2005) Successful myomectomy during pregnancy: A case report. Reprod Health 2: 6.

9. Carolis SD, Fatigante G, Ferrazzani S, Trivellini C, Santis LD, et al. (2011) Uterine myomectomy in pregnant women. Fetal Diagn Ther 16: 116-119.

10. Celik C, Acar A, Nedim C, Kazim G, Cemalettin A (2002) Can myomectomy be performed during pregnancy? Gynecol Obstet Invest 53: 79-83.
11. Fanfani F, Rossitto C, Fagotti A, Rosati P, Gallotta V, et al. (2010) Laparoscopic myomectomy at 25 weeks of pregnancy: case report. J Minim Invasive Gynecol 17: 91-93.

12. Bhatla N, Dash BB, Kriplani A, Agarwal N (2009) Myomectomy during pregnancy: a feasible option. J Obstet Gynaecol Res 35: 173-175.

13. Kaser O, Ikle FA, Hirsch HA (1985) Surgery on the Uterus: Atlas of Gynecological Surgery (2ndedn), Thieme, Stuttgart, Germany. 7.1-7.29.

14. Makar AP, Schatteman EA, Vergote IB, Desmedt E (1989) Myomectomy during pregnancy: uncommon case report. Acta Chir Belg 89: 212-214.

15. Mollica G, Pittini L, Minganti E, Perri G, Pansini F (1996) Elective uterine myomectomy in pregnant women. Clin Exp Obstet Gynecol 23: $168-172$

16. Leite GK, Korkes HA, Viana Ade T, Pitorri A, Kenj G, et al. (2010) Myomectomy in the second trimester of pregnancy: case report. Rev Bras Ginecol Obstet 32: 198-201.

17. Sheiner E, Bashiri A, Levy AJ, Hershkovitz R, Katz M, et al. (2004) Obstetric characteristics and perinatal outcome of pregnancies with uterine leiomyomas. J Reprod Med 49: 182-186.

18. Zaima A, Ash A (2011) Fibroid in pregnancy: characteristics, complications and management. Postgrad Med J 87: 819-828.

19. Radhika BH, Naik K, Shreelatha S, Vana H (2015) Case series: Pregnancy Outcome in Patients with Uterine Fibroids. J Clin Diagn Res 9: QR01-QR04.

20. Gaffney A (2014) Critical care in pregnancy - is it different? Semin Perinatol 38: 329-340.

21. Clark V, Fernando R, Van de Velde M (2016) Oxford Textbook of Obstetric Anaesthesia, Oxford University Press, USA.

22. Lapinsky SE (2015) Endotracheal intubation in the ICU. Crit Care 17: 258.

23. Lapinsky SE, Kruczynski K, Slutsky AS (1995) Critical care in the pregnant patient. Am J Respir Crit Care Med 152: 427-455.

24. Cole DE, Taylor TL, McCullough DM, Shoff CT, Derdak S (2005) Acute respiratory distress syndrome in pregnancy. Crit Care Med 33: S269-S278.

25. Bedson R, Riccoboni A (2014) Physiology of pregnancy: clinical anaesthetic implications. Contin Educ Anaesth Crit Care Pain 14: 69-72.

26. Clardy PF, Reardon CC (2017) Critical illness during pregnancy and the peripartum period. 